\title{
ANÁLISE DA CAPACIDADE FUNCIONAL DE IDOSOS INSTITUCIONALIZADOS Á PARTIR DA AUTO-AVALIAÇÃO
}

\author{
Daniel Vicentini de Oliveira \\ Márcia Regina Benedeti \\ Rosangela Cocco Morales \\ Terezinha Gomes Faria
}

\section{Resumo}

Este estudo teve como objetivo analisar a capacidade funcional para a realização das atividades da vida diária (AVDs) de idosos residentes nas instituições assistenciais do município de Maringá/PR, bem como determinar a média de idade, prevalência de gênero, capacidade funcional correlacionada com a idade cronológica e com o gênero, assim como com o tempo de institucionalização. O instrumento utilizado foi o protocolo intitulado "Ficha de Auto-Avaliação da Capacidade Funcional" composto por 18 itens que avaliam a capacidade do indivíduo em realizar as tarefas básicas do cotidiano de um idoso. O estudo foi desenvolvido em três instituições asilares filantrópicas do município de Maringá-PR, com 70 idosos. Deste total, 40 do gênero masculino e 30 do feminino, com idade variando entre 60 e 101 anos, no período compreendido entre Agosto e Outubro de 2008. Obteve-se como resultados um alto índice de dependência na realização das atividades da vida diária, sendo a capacidade funcional classificada como baixa $(47 \%)$ ou moderada (49\%). O trabalho revelou ainda um baixo índice de independência total (4\%) além da importância da participação de profissionais da área da saúde devidamente habilitados. Desta forma, estes poderão auxiliar nas limitações da capacidade funcional buscando a prevenção e a reabilitação precoce.

\section{Palavras-Chave}

Idosos; Capacidade funcional; Asilo

\section{ANALYSIS OF THE FUNCTIONAL CAPACITY OF ELDERLY INSTITUCIONALIZED FROM THE AUTO EVALUATION}

Daniel Vicentini de Oliveira

Márcia Regina Benedeti

Rosangela Cocco Morales

Terezinha Gomes Faria

\begin{abstract}
This study had the objective to analyze the functional capacity for the accomplishment of the daily life activities (DLAs) of elderly, that are residents in the assistance institutions of the city of Maringa/PR, as well as to determinate the average of age, prevalence of gender, functional capacity correlated with the chronological age and the gender, just like the institutionalization time. The used instrument was the entitled protocol "Ficha de Auto-Avaliação da Capacidade Funcional" composed with 18 items that evaluate the individual capacity of realizing the basic daily life tasks of one elder. The study was developed in three philanthropic asylum institutions of the city of Maringá-PR, with 70 elderly. From this total 40 of feminine gender and 30 of masculine gender, with the age varying between 60 and 101 years
\end{abstract}


old, in an understood period between August and October of 2008. It was gotten as results one high index of dependence in the accomplishment of the daily life activities, being the functional capacity classified as low $(47 \%)$ or moderate $(49 \%)$. The study even showed a low index of total independence $(4 \%)$ besides the importance of the participation of the health area professionals duly qualified. This way, they will be able to assist in the limitations of the functional capacity searching for prevention and early rehabilitation.

\section{Key-Words}

Elder, Functional capacity, Asylum 


\section{INTRODUÇÃO}

No Brasil, em 1960, o número de idosos alcançava três milhões de pessoas, sete milhões em 1975, dez milhões e meio em 1991, quatorze milhões em 2002 e estima-se que poderá alcançar a faixa dos trinta e dois milhões em 2020 (UENO, 1999).

Nos países em desenvolvimento, os indivíduos acima de 60 anos são os "participantes" da terceira idade, e nos países desenvolvidos, acima dos 65 anos (OLIVEIRA; SILVA; KRAVCHYCHYN, 2007).

Muitas são as teorias e explicações sobre o envelhecimento do ser humano: A teoria do envelhecimento programado, a teoria do encurtamento do telômero, a teoria da mutação somática, a teoria da catástrofe do erro, a teoria do envelhecimento auto-imune, a teoria do lixo celular, entre outras (HAMILTON, 2002).

As alterações a que os idosos são submetidos são consideradas naturais, gradativas e gerais, ou seja, pode-se verificá-las em idade mais precoce ou mais avançada e em maior ou menor grau, de acordo com as características genéticas de cada um. É também um processo universal e irreversível, que se acelera na maturidade e provoca uma perda progressiva no organismo (ZIMERMAN, 2000; HAMILTON, 2002)

Gallahue e Ozmun (2003) salientam que a massa muscular diminui á medida que o número e o tamanho das fibras musculares declinam durante o final da meia idade e dos anos posteriores da idade adulta. Funcionalmente, uma diminuição na força muscular parece ocorrer ao mesmo tempo em que ocorre essa perda no tecido muscular. Segundo o autor, por volta dos sessenta anos há uma perda aproximada de vinte por cento da força e uma perda mais acentuada, de $40 \%$, entre 70 e 80 anos.

Observam-se também alterações no sistema cardíaco, nervoso, respiratório e nos cinco sentidos com o processo de envelhecimento (GUCCIONE, 2000; HAMILTON, 2002, GALLAHUE; OZMUN, 2003).

No aspecto psicológico, pode-se citar a dificuldade de se adaptar a novos papéis, a falta de motivação e a dificuldade de planejar o futuro, a dificuldade de se adaptar às mudanças rápidas, depressão, hipocondria, somatização, paranóia, tentativas de suicídios, comprometimento da auto-imagem e auto-estima, aceitação ou recusa da situação de velho, aceitação ou rejeição do meio, diminuição da vontade, das 
aspirações e da atenção, apego ao conservadorismo, desconfiança, irritabilidade e indocilidade (MEIRELLES, 1997; ZIMERMAN, 2000).

Zimerman (2000) complementa com as modificações sociais que podem ocorrer no indivíduo na terceira idade, em constante etapa de envelhecimento: crise de identidade provocada pela falta de papel social, o que levará o individuo a uma perda de sua auto-estima; mudanças de papeis na família, no trabalho e na sociedade; aposentadoria, perdas diversas, diminuição dos contatos sociais, entre outras alterações.

Com o acelerado crescimento mundial da população idosa, a preocupação em relação à capacidade funcional vem surgindo como novo destaque, pois á medida que se envelhece muitas tarefas do cotidiano vão tornando-se cada vez mais difíceis de serem realizadas (ROSA et al., 2003; ARAÚJO; CEOLIM, 2007).

Ueno (1999), define capacidade funcional como a capacidade de realizar as atividades da vida diária independentemente, incluindo as atividades de deslocamento, de auto-cuidado, sono adequado e participação em atividades ocupacionais e recreativas.

A capacidade funcional é avaliada a partir das AVDs ( atividades básicas da vida diária) e AIVDs (atividades instrumentais da vida diária). As AVDs são atividades de cuidado pessoal que a própria pessoa pode realizar todos os dias, como comer, vestir-se, tomar banho, realizar transferência de posições, controlarem a bexiga e intestino. Já as AIVDs são atividades que possibilitam qualquer pessoa a viver com independência em sua casa ou apartamento, como preparar refeições, realizar atividades caseiras, tomar medicamentos, usar telefone, realizar atividades financeiras (REBELATTO; MORELLI, 2007).

A incapacidade funcional é uma condição multifatorial que difere em relação a: causas, natureza, forma de aparecimento, ritmo e implicações sociais, consistindo mais em um processo do que em um estado estático (GIACOMIN et al., 2008).

Verbrugge e Jette (1994) citados por Gobbi et al. (1008) propõem um modelo teórico do processo de tornar-se incapaz, considerando três aspectos: (I) fatores predisponentes (características 
sóciodemográficas); (II) fatores intra-individuais (estilo de vida, atributos psicossociais, mudanças de comportamento, maneiras de lidar com as dificuldades, com as doenças e com as modificações de atividades que podem afetar o processo de incapacidade); e (III) extra-individuais (intervenções dos serviços de saúde e de reabilitação, uso de medicamentos, suportes externos e ambiente físico e social).

Assim, é comum o idoso depender de cuidados especiais, de cuidadores ou familiares para a realização de tarefas muito comuns, como alimentar-se, vestir-se, tomar banho ou até mesmo realizar os hábitos de higiene e necessidades pessoais (MINCATO; FREITAS, 2007).

Enfim, o envelhecimento traz consigo inúmeras modificações no organismo físico, psicológico e social, que resultam na diminuição do desempenho motor, principalmente na realização das atividades da vida diária (AVD's), o que pode, ou não, levar esses indivíduos a se tornarem dependentes de outros (ANDREOTTI; OKUMA, 1999).

Quadros Júnior (2008) cita que como conseqüência dessa diminuição na realização das atividades da vida diária, é provável um aumento do número de idosos residentes em instituições de longa permanência para idosos, popularmente conhecidas por asilos.

Segundo o Decreto $\mathrm{n}^{\mathrm{o}}$ 1.948, de 03 de Julho de 1996, artigo $3^{\circ}$, existem formas distintas de atendimento aos idosos: a modalidade asilar, definida como atendimento, em regime de internato, ao idoso sem vínculo familiar ou sem condições de prover a própria subsistência, de modo a satisfazer as suas necessidades de moradia, alimentação, saúde e convivência social, que rege a vida do mesmo por meio de normas específicas, e o ampara civil e socialmente e a modalidade não asilar, que se compõe de Centro de Convivência, Centro de Cuidados Diurno, Hospital-Dia, Casa-Lar e Oficina Abrigada de Trabalho e destina-se a atender o idoso por determinado período do dia (YAMAMOTO; DIOGO, 2002; MINCATO; FREITAS, 2007).

Mincato e Freitas (2007), em seu estudo, afirmam que grande parte das entidades de atendimento aos idosos são instituições religiosas e filantrópicas, que se mantêm por meio de doações e que tem como função social abrigar os idosos com problemas de moradia, sem família e carentes de recursos econômicos para sua subsistência. 
Prioriza, também, a Lei 8.842, de janeiro de 1994, no artigo $4^{\circ}$, parágrafo III, atendimento ao idoso pelas famílias, ao invés do asilar (DAVIM et al., 2004).

Define-se asilo (do grego, ásylos, pelo latim asylu) como casa de assistência social onde são recolhidas para sustento ou também para educação, pessoas pobres e desamparadas, como mendigos, crianças abandonadas, órfãos e velhos. Relaciona-se assim, a idéia de guarita, abrigo, proteção ao local denominado asilo, independentemente do seu caráter social, político ou de cuidados com dependências físicas e/ou mentais. Eles são a modalidade mais antiga e universal de atendimento ao idoso fora do convívio familiar, porém, apresentam fatores negativos, como o isolamento social e a inatividade física e mental (GORZONI; PIRES, 2006)

O estatuto do idoso prevê algumas obrigações a essas entidades de atendimento ao idoso, dentre elas, cuidados à saúde; promoção de atividades educacionais, esportivas, culturais e de lazer e manter no quadro de funcionários, profissionais com formação especifica. A instituição asilar tem o compromisso de suprir as necessidades básicas dos idosos, proporcionando-lhes uma melhor qualidade de vida. Contudo, nem sempre lhes são oferecidas atividades, por falta de mão-de-obra especializada, problemas financeiros ou até mesmo pela restrição de espaço físico. Assim, os idosos ficam muito tempo ociosos, o que pode levar a problemas de angústia e depressão, entre outras doenças (MINCATO; FREITAS, 2007).

Segundo Born (2002) os idosos ao ingressarem nos asilos começam a apresentar limitações intelectuais e físicas que se tornam evidentes na realização das atividades da vida diária, sendo que o ócio, a falta de terapia ocupacional, a indisposição física e o desinteresse, colaboram ainda mais para estas limitações, levando muitas vezes á invalidez e ao profundo abatimento moral, surgindo assim ás doenças crônicodegenerativas associadas á outras patologias, que podem ser responsáveis pela perda progressiva de autonomia.

Os idosos que vivem em asilos, casas geriátricas e clínicas apresentam características importantes como aumento de sedentarismo, perda de autonomia, ausência de familiares, que entre outros, contribuem para o aumento das prevalências das morbidades e co-morbidades relacionadas à autonomia (GONÇALVES et al., 2008). 
$\overline{\text { Estes idosos apresentam nível de atividade física médio muito baixo - inferior a } 1,0 \text { ponto, quando }}$ avaliados pelo Questionário Baecke Modificado para Idosos (VOORRIPS; RAVELLI; DONGELMANS; DEURENBERG; VAN STAVEREN, 1991 citado por CORAZZA, 2005). Nesse sentido, é provável que a institucionalização do idoso seja altamente relacionada também com o declínio cognitivo (MOLASCHI, SCARAFIOTTI et al., 1998 citado por GOBBI et al., 2008) e com baixos níveis de atividade física.

Araújo e Ceolim (2007) ressaltam que a avaliação da capacidade funcional é relevante em gerontologia, como indicativo de qualidade de vida do idoso. O desempenho das atividades da vida diária é considerado um parâmetro aceito e legítimo para firmar essa avaliação, sendo utilizado pelos profissionais da área da saúde, para avaliar graus de dependência de seus clientes.

Com isso, este estudo teve como objetivo analisar a capacidade funcional para a realização das atividades da vida diária (AVDs) de idosos residentes nas instituições assistenciais do município de Maringá/PR, bem como determinar a média de idade, prevalência de gênero, capacidade funcional correlacionada com a idade cronológica, com o gênero, assim como com o tempo de institucionalização.

\section{METODOLOGIA}

Trata-se de uma pesquisa descritiva, observacional e transversal. Antes da coleta de dados, o projeto foi encaminhado para análise e parecer do Comitê Permanente de Ética em Pesquisa do Cesumar (COPEC) e aprovada no dia 07 de Julho de 2008 com o protocolo número 055/2008, segundo a Resolução 196/96, do Conselho Nacional de Saúde, que dispõe sobre pesquisa realizada com seres humanos. Após aprovação, os responsáveis pelas instituições foram consultados sobre a pesquisa e a seguir, as que aceitaram assinaram um Termo de Consentimento Livre e Esclarecido.

A população do estudo constitui-se de 165 , residentes em três instituições públicas de assistência domiciliar ao idoso do município de Maringá-PR. Essas instituições foram caracterizadas como A, B e C com a finalidade de manter o seu sigilo e de seus residentes.

Foram excluídos da amostra idosos não cadastrados como residentes em uma das instituições estudadas, idosos que apresentavam incapacidade funcional (restritos ao leito, usuários de cadeia de rodas), portadores de paralisia cerebral ou com comprometimento cognitivo, idosos com seqüelas graves de acidente vascular encefálico, epilepsia, multinfarto, idosos com diminuição grave da audição, afasia, 
dificuldade de fala e expressão. Foram inclusos idosos com idade igual ou superior á sessenta anos, residentes, cadastrados nas instituições avaliadas e com capacidade de expressão, fala e compreensão preservadas.

A amostra foi composta por 70 idosos, sendo 40 do gênero masculino e 30 do gênero feminino.

O instrumento utilizado foi o protocolo intitulado "Ficha de Auto-Avaliação da Capacidade Funcional" de Spirduso (1994) citado por Matsudo (2005) composto por 18 itens para avaliar a capacidade do indivíduo em realizar determinadas tarefas. A coleta dos dados foi realizada pelo pesquisador que questionava oralmente o idoso e obtinha como resposta uma das alternativas oferecidas. Isso explica a necessidade da exclusão de idosos que não tinham capacidade de audição, fala e compreensão preservada.

As 18 atividades analisadas são categorizadas em: 1)- realiza sem ajuda e com facilidade, 2)- realiza sem ajuda, mas com algum grau dificuldade ou 3)- realiza com ajuda ou depende de outros para realizar. Posteriormente foram analisados e somados os escores. Como resultado, podia-se obter: capacidade funcional baixa (realiza 0 a 9 atividades classificadas na categoria 1); capacidade funcional moderada (realiza de 10 a 17 atividades classificadas na categoria 1) e capacidade funcional avançada (realiza as 18 atividades classificadas na categoria 1$)$.

A análise dos dados foi pela estatística descritiva, e os resultados dispostos sob forma de tabelas, através de média, freqüência, percentual, desvio padrão e a utilização dos testes t-Student, Qui-quadrado e Correlação de Pearson, todos com significância de p>0,05.

\section{RESULTADOS}

Serão apresentados os resultados referentes á amostra das instituições que estão identificadas pelas letras A (24 idosos - 34\%), B (17 idosos - 24\%) e C (29 idosos - 42\%) no total de setenta (70) idosos institucionalizados.

Os dados obtidos confirmam os dados apresentados pela literatura onde as instituições possuem características semelhantes, pois também se tratam de instituições filantrópicas e são mantidas por promoções e doações realizadas pela comunidade. A instituição B é voltada ao atendimento de idosos japoneses e descendentes. 
Dos 70 indivíduos analisados, observou-se uma prevalência do sexo masculino, com 40 (57\%) homens e 30 (43\%) mulheres. A instituição A, constou com 14 homens (58\%) e 10 mulheres (42\%) do total de 24 idosos; a instituição B, com 10 homens (59\%) e 07 mulheres (41\%) do total de 17 e a instituição C, com 14 homens (48\%) e 15 mulheres (52\%) do total de 29.

Vários estudos realizados em instituições semelhantes, em cidades dos estados do Rio Grande Sul, São Paulo, Paraíba e Rio de Janeiro confirmam a prevalência do gênero feminino (BENTO, 1999; BENNEMANN, 2002 apud GUEDES; SILVEIRA, 2004; OLIVEIRA; SOUZA; FREITAS, 2006; ARAUJO; CEOLIM, 2007; MINCATO; FREITAS, 2007).

Os dados das pesquisas desses autores confirmam a tendência do gênero feminino onde a participação masculina raramente ultrapassa os 20\% (DAVIM et al., 2004).

Porém os dados recolhidos nas instituições de Maringá/PR, em relação ao gênero são inversos se comparados aos estudos de Bento e Bennemann (1999, 2002) apud Guedes e Silveira (2004) e de Oliveira, Souza e Freitas (2006), Aráujo e Ceolim (2007) e Mincato e Freitas (2007). Como causa, poderíamos supor que cada região possui as suas características físicas, culturais e étnicas, e que a prevalência de mulheres idosas no Brasil, não é geral para todos os estados e municípios, portanto em Maringá, ao norte do estado do Paraná, nas três instituições avaliadas prevaleceram os sujeitos do gênero feminino.

A idade média dos idosos foi de 75,5 anos, com desvio padrão de 8,8, variando entre 60 e 101 anos. Obteve-se um predomínio na faixa de idade entre 71 e 80 anos, com uma freqüência de 31 idosos (44\%). Entre 60 e 70 anos, obteve-se 17 idosos (24\%); entre 81 e 90 anos, 19 idosos (28\%) e 03 indivíduos (4\%) com mais de 90 anos.

Na literatura, obtiveram-se dados semelhantes. Na pesquisa de Savonitti (2000) apud Guedes e Silveira (2004) a idade média foi de 73,1 anos e 78,3 anos no estudo de Mincato e Freitas (2007). Em relação a faixas etárias a pesquisa de Araújo e Ceolim (2007) obteve como resultado que a maioria dos sujeitos tinha idade entre 70 e 79 anos (38\% do total), e o de Oliveira, Souza e Freitas (2006) verificou que a 
maior parte dos idosos possuía também idade entre 70 e 79 anos (30,3\% do total), semelhante aos dados coletados na presente pesquisa.

Com relação ao tempo de internação (TABELA 1), 06 idosos (9\%) estão institucionalizados á menos de 1 ano; 47 idosos (67\%) no intervalo de 1 a 10 anos; seguido de 10 idosos (14\%) institucionalizados de 11 a 20 anos; 5 idosos (7\%) no intervalo de 21 a 30 anos e somente 2 idosos (3\%) a mais de 30 anos. $\mathrm{O}$ tempo médio de institucionalização foi de 6,7 anos, com desvio padrão de 7,5.

TABELA 1 - Tempo de institucionalização dos idosos

\begin{tabular}{lcc}
\hline \multirow{2}{*}{ INSTITUCIONALIZAÇÃO } & \multicolumn{2}{c}{ FREQUÊNCIA } \\
\cline { 2 - 3 }$<1$ ano & $\mathbf{N}$ & $\mathbf{\%}$ \\
$1-10$ anos & 47 & 9 \\
$11-20$ anos & 10 & 67 \\
$21-30$ anos & 5 & 14 \\
$>30$ anos & 2 & 7 \\
TOTAL & $\mathbf{7 0}$ & $\mathbf{1 0 0}$ \\
\hline
\end{tabular}

De acordo com o estudo de Lucena et al. (2002) apud Guedes e Silveira (2004), o tempo médio de residência foi de 7,82 anos, sendo o idoso institucionalizado há mais tempo com 25 anos e o mais recente, há apenas um ano.

É considerável o número de idosos que moram em instituições há mais de 10 anos, o que favorece sua exclusão social do ponto de vista citadino. Nesses casos, mesmo que a instituição atenda às necessidades básicas do idoso, a mesma não se apresenta como sendo o ambiente mais adequado para que eles envelheçam, pois se tornam limitados ao convívio entre si, não participando da sociedade em sua totalidade política, produtiva e cultural.

No trabalho de Reis, Torres e Reis (2008), dos 60 idosos estudados, na cidade de Jequié/BA, 85\% morava no asilo no intervalo de 1 a 10 anos, dado esse semelhante ao presente estudo.

Utilizando como instrumento de avaliação a Ficha de Auto Avaliação da Capacidade Funcional, de Matsudo (2005), pode-se analisar a prevalência de incapacidade funcional, dificuldades e autonomia na 
realização das AVD's de cada indivíduo, permitindo portanto a classificação da capacidade funcional em avançada moderada e baixa.

Conforme a Tabela 2, dos 70 indivíduos avaliados, somente 03 (4\%) consegue realizar todas as atividades da vida diária com facilidade e autonomia; 34 (49\%) realizam de dez a dezessete atividades e 33 (47\%) realizam nenhuma ou até nove atividades da vida diária com autonomia e facilidade, tendo dificuldades na realização das outras e/ou precisando da ajuda de algo ou alguém.

\section{TABELA 2 - Porcentagem de idosos em relação à capacidade funcional para realização das AVD's com autonomia e facilidade, conforme a Ficha de Auto Avaliação da Capacidade Funcional ${ }^{1}$}

\begin{tabular}{lcc}
\hline & \multicolumn{2}{c}{ FREQUÊNCIA } \\
\cline { 2 - 3 } CLASSIFICAÇÃO AVDs & N & \% \\
\hline Baixa & 33 & 47 \\
Moderada & 34 & 49 \\
Avançada & 3 & 4 \\
TOTAL & $\mathbf{7 0}$ & $\mathbf{1 0 0}$ \\
\hline
\end{tabular}

${ }^{1}$ Fonte: MATSUDO, 2005.

Araújo e Ceolim (2007) verificaram que, dos 187 sujeitos institucionalizados pesquisados, 70 deles (37\%) podiam ser considerados independentes para o desempenho das AVD's. No estudo de Yamamoto e Diogo (2002) na cidade de Campinas-SP, em três instituições pesquisadas, houve o predomínio de indivíduos parcialmente dependentes e em outras três, de independentes. Esses dados apontam a necessidade da contratação de pessoas qualificadas para a assistência.

Quanto á prevalência de incapacidades em relação ao gênero obteve-se que, dos três indivíduos com capacidade funcional avançada, todos eram do gênero masculino. Dos 34 idosos com capacidade funcional moderada, 18 eram do gênero masculino e 16 do feminino, e dos 33 com capacidade funcional baixa, 19 eram do gênero masculino e 14 do feminino.

Observou-se no presente estudo que a maioria dos idosos do gênero masculino apresentava capacidade funcional baixa.

Ao contrário, no estudo de Mincato e Freitas (2007), a capacidade funcional dos homens apresentou-se maior e melhor do que das mulheres; porém, no de Araújo e Ceolim (2007), do total de 70 idosos 
independentes, em três instituições, 59 eram mulheres e 11 eram homens, ou seja, as mulheres realizavam suas atividades da vida diária com autonomia e no de Guedes e Silveira (2004), as mulheres aparecem sendo também mais independentes que os homens, com $61 \%$ contra $58 \%$ respectivamente.

Nota-se que a capacidade funcional de idosos institucionalizados não se atém somente a um gênero pois de acordo com o teste do "Qui quadrado", obteve-se que não possui significância com a capacidade funcional $(\mathrm{p}=0,573)$, se considerarmos capacidade avançada e moderada como normal e capacidade funcional baixa como anormal. A capacidade funcional varia, independentemente do gênero, o que sugere a influência do estilo de vida sedentário adotado por eles e a falta de condições das instituições em oferecer aos idosos momentos diários de atividade física.

Dos 17 idosos com idades entre 60 e 70 anos, $70 \%$ foram avaliados como tendo capacidade funcional baixa, realizando somente de nenhuma á nove atividades da vida diária sem ajuda e com facilidade, tendo dificuldades nas demais e/ou necessitando da ajuda de algo ou alguém. Dos 31 idosos com idades entre 71 e 80 anos, (33\%) avaliaram-se com capacidade funcional baixa; dos 19 com idades entre 81 e 90 anos (47\%) avaliaram-se com capacidade funcional baixa e dos 3 com mais de 90 anos (67\%) avaliaram-se com capacidade funcional baixa (TABELA 3)

TABELA 3 - Capacidade funcional dos idosos institucionalizados de acordo com a faixa etária

\begin{tabular}{lcccccc}
\hline & \multicolumn{5}{c}{ CAPACIDADE FUNCIONAL } \\
\cline { 2 - 7 } & \multicolumn{3}{c}{ AVANÇADA } & \multicolumn{1}{c}{ MODERADA } & BAIXA \\
\hline IDADE & N & $\mathbf{~ \% ~}$ & N & \% & N & $\%$ \\
\hline $60-70$ anos & 01 & 5 & 04 & 23 & 12 & 70 \\
$71-80$ anos & 01 & 3 & 20 & 64 & 10 & 33 \\
$81-90$ anos & 01 & 5 & 09 & 47 & 09 & 47 \\
$>90$ anos & 00 & 0 & 01 & 33 & 02 & 67 \\
TOTAL & $\mathbf{0 3}$ & & $\mathbf{3 4}$ & & $\mathbf{3 3}$ & \\
\hline
\end{tabular}

Percebeu-se que a maioria dos idosos com capacidade funcional classificada como baixa se encontrava no intervalo de idades entre 60 e 70 anos, e não nas idades mais avançadas como relata alguns estudos (COSTA et al. apud GUEDES; SILVEIRA, 2004; MINCATO; FREITAS, 2007).

Observou-se que os estágios iniciais da velhice, entre 60 e 70 anos, e o estágio final, mais de 90 anos, são os que apresentam porcentagem maior de indivíduos com capacidade funcional baixa, e o estágio 
intermediário, de 71 á 80 anos, apresentou a maior porcentagem de indivíduos com capacidade funcional moderada.

Ao analisar os dados obtidos, é relevante salientar que os indivíduos que se encontram com capacidade funcional moderada, com o passar do tempo e com a vida sedentária que levam, podem decair para uma capacidade funcional baixa, passando, portanto a depender de outros para realização da maioria das atividades da vida diária.

Uma correlação foi realizada entre a capacidade funcional dos indivíduos em realizar pelo menos uma atividade sem dificuldade e sem ajuda, com a idade de cada idoso. Obteve-se uma correlação fraca $(\mathrm{r}=0,014)$, com forte indicativo de que é inviável afirmar que quanto maior a idade do individuo, menor será sua capacidade funcional de realizar as atividades com autonomia e facilidade.

Os sujeitos internados há menos de sete meses foram excluídos, pois não foram encontrados dados consistentes na literatura no que diz respeito a esse aspecto. Conforme a Tabela 4 teve-se como resultado que os indivíduos institucionalizados entre sete meses e onze meses, apresentam prevalência de capacidade funcional moderada (75\%). Indivíduos com tempo de institucionalização entre 1 e 10 anos, apresentavam em sua maioria capacidade funcional baixa (56\%); de 11 a 20 anos, capacidade funcional moderada (80\%), de 21 a 30 anos, capacidade funcional moderada $(60 \%)$ e com mais de 30 anos de institucionalização, apenas dois indivíduos, um apresentando capacidade funcional moderada e a outra baixa.

TABELA 4 - Classificação da capacidade funcional dos idosos em relação ao tempo de institucionalização

\begin{tabular}{llclclll}
\hline & \multicolumn{5}{c}{ CAPACIDADE FUNCIONAL } \\
\cline { 2 - 7 } & AVANÇADA & & MODERADA & & BAIXA & \\
\hline Tempo de Internação & N & $\%$ & N & \% & N & \% \\
\hline 7 meses -11 meses & 0 & 0 & 3 & 75 & 1 & 25 \\
$1-10$ anos & 2 & 4 & 18 & 39 & 26 & 56 \\
$11-20$ anos & 0 & 0 & 8 & 80 & 2 & 20 \\
$21-30$ anos & 0 & 0 & 3 & 60 & 2 & 40 \\
$>30$ anos & 0 & 0 & 1 & 50 & 1 & 50 \\
\hline
\end{tabular}

Após a análise dos dados pode-se perceber que os indivíduos da instituição $\mathrm{B}$, na sua totalidade de etnia japonesa, possuem uma capacidade funcional para realização das atividades diárias mais avançadas em Conexões: revista da Faculdade de Educação Física da UNICAMP, Campinas, v. 7, n. 2, p. 79-96, maio/ago. 2009. 
comparação aos indivíduos das outras duas instituições. Por isso, foram comparados estatisticamente os grupos das instituições $\mathrm{A}$ e $\mathrm{C}$ com os da instituição $\mathrm{B}$, através do teste t-Student, mostrando que há uma elevada significância estatística na capacidade funcional dos indivíduos da instituição $B(p=0,0005)$, ou seja, nesta instituição encontram-se idosos com capacidade funcional preservada, portanto realizam suas atividades de maneira independente e sem maiores dificuldades.

Correlacionando a instituição B e a $C$, obteve-se significância estatística altamente relevante $(p=0,006)$ para capacidade funcional dos indivíduos da instituição B, o mesmo encontrou-se quando comparados a instituição A com a B ( $\mathrm{p}=0,0005)$.

Finalmente, correlacionou-se as instituições A e C, obtendo-se uma não significância estatística $(\mathrm{p}=0,325)$ para capacidade funcional dos idosos pertencentes as instituições referidas, demonstrando que em ambas seus idosos apresentam praticamente a mesma capacidade funcional para realização da maioria das atividades da vida diária.

Um estudo de Matsubayashi, Ishine, Wada e Okumiya (2006) apud Teixeira e Neri (2008) realizado em 5.207 cidades do Japão, relatou que um dos itens importantes para um envelhecimento bem sucedido era manter a capacidade de auto-cuidado até próximo da morte. Porém esses são dados de pesquisas com populações de mesma etnia dos idosos da instituição $\mathrm{B}$, mas de localidade e região diferentes do presente estudo, ao mesmo tempo em que isso é comprovado não ser aplicado a todos os indivíduos da etnia japonesa.

\section{DISCUSSÃO DOS RESULTADOS}

Os dados obtidos no presente estudo indicam uma baixa capacidade funcional dos idosos residentes nas instituições, surgindo, portanto a necessidade de intervenções.

Confirmando resultados semelhantes na literatura, a maioria dos idosos institucionalizados apresentava-se por volta dos 70 anos de idade, faixa etária com o maior número de idosos com capacidade funcional moderada. O número de idosos com capacidade funcional avançada é baixo, e prevaleceu igualmente entre as idades de 60-70 anos, 71-80 anos e surpreendentemente na faixa dos 81-90 anos o que vai de encontro à literatura, que o envelhecimento é um processo gradual, irreversível, porém é individual, ou seja, cada um envelhece de acordo com seu sistema orgânico hereditário, independente da idade. Os 
idosos com prevalência de capacidade funcional baixa apresentavam-se no intervalo de 60 á 70 anos de idade, e não em idades mais avançadas, que era o de se esperar, e o que é comprovado na maioria dos estudos.

Através da correlação de Pearson, realizada entre a capacidade funcional dos indivíduos em realizar pelo menos uma atividade sem dificuldade e sem ajuda, com a idade de cada um, obteve-se uma correlação fraca, mostrando que não podemos afirmar que quanto maior a idade do individuo, menor será sua capacidade funcional de realizar as atividades com autonomia e facilidade.

Em relação ao gênero, obteve-se uma maior prevalência de indivíduos do gênero masculino, e que apresentam maior incapacidade funcional, ou seja, realizam somente de uma á nove atividades sem ajuda e com facilidade, ou não realizam. No entanto, não houve relação estatisticamente significativa da variável gênero com o grau de capacidade funcional.

Analisando a capacidade funcional, com o tempo de permanência nas instituições, os dados relataram que a maioria dos indivíduos com capacidade funcional baixa e moderada se encontravam institucionalizados no intervalo de 1 a 10 anos e não nos intervalos de tempo maiores.

No andamento da pesquisa, pode-se perceber que os idosos de descendência ou origem japonesa, apresentavam maior autonomia e/ou facilidade na execução da maioria das atividades da vida diária. $\mathrm{Na}$ análise dos dados, através do teste "t-Student", ao comparar esses indivíduos (instituição B) com as outras instituições (A e C), que possuíam idosos de diversas descendências, mostrou-se uma elevada significância estatística na capacidade funcional dos idosos japoneses em relação aos demais. Estudos nessa área são escassos.

\section{CONCLUSÃO}

Diante desses dados pode-se concluir sobre a importância da participação de profissionais da área da saúde, devidamente capacitados e habilitados que poderão auxiliar nas limitações da capacidade funcional, buscando a prevenção, reabilitação precoce, prevenindo, portanto a perda funcional. Sabe-se que a tendência nos próximos anos é de um aumento expressivo, inclusive nas instituições de cuidado ao idoso, o que nos remete a pensar, discutir, refletir e encontrar formas alternativas de integração social, convívio familiar e, sobretudo, de prevenção do declínio da capacidade funcional. O profissional da 
saúde, por meio da atividade física, consegue alcançar muitos desses objetivos, favorecendo uma velhice bem sucedida, dentro dos padrões de dignidade humana e da boa qualidade de vida

Há ainda, a necessidade de ampliar e aprofundar pesquisas com avaliações que englobem o idoso em todas as dimensões (por instrumentos mais abrangentes em relação a sua saúde física e mental) e também avaliar o ambiente físico no qual ele está inserido, pois ambientes; inadequados contribuem para a diminuição da capacidade funcional. Dados referentes a essas variáveis serão coletados em trabalhos futuros para que se possam implementar medidas e ações no que diz respeito à capacidade funcional dos idosos moradores das instituições prestadoras desse gênero de serviços.

\section{REFERÊNCIAS}

ANDREOTTI, R.A; OKUMA, S.S. Validação de uma bateria de testes de atividades da vida diária para idosos fisicamente independentes. Rev Paul Educ Fis, n.13,1999.

ARAUJO, M. O. D. H; CEOLIM, M. F. Avaliação do grau de independência de idosos residentes em instituições de longa permanência. Rev Esc Enferm USP, v. 41, n. 3, set. 2007.

BORN, T. Cuidado ao idoso em instituição. In: NETTO, M. P. (Org.). Gerontologia: a velhice e o envelhecimento em visão globalizada. São Paulo: Atheneu, 2002. p. 1131-1141

DAVIM, R. M. B. et al. Estudo com idosos de instituições asilares no município de Natal/RN: características socioeconômicas e de saúde. Rev Latino Americana Enferm, v. 12, n. 3, maio/jun. 2004.

GAllahUE, D. L.; OZMUN, J. C. Compreendendo o desenvolvimento motor: bebês, crianças, adolescentes e adultos. São Paulo: Phorte, 2001

GIACOMIN, K.C et al. Estudo de base populacional dos fatores associados à incapacidade funcional entre idosos na Região metropolitana de Belo Horizonte/MG, Brasil. Cad Saúde Pública, v. 24, n. 6, p.1260-1270, 2008.

GOBBI, S. et al. Comportamento e barreiras: atividade física em idosos institucionalizados. Psicol: teoria e pesquisa, v. 24, n. 4, p. 451-458, 2008.

GONÇALVES, L. G. et al. Prevalência de quedas em idosos asilados do município de Rio Grande/RS. Rev Saúde Pública, v.42, n.5, p.938-945, 2008.

GORZONI, M. L; PIRES, S. L. Aspectos clínicos da demência senil em instituições asilares. Rev Psquiatr Clin USP, São Paulo, v. 33, n. 1, p. 18-23, 2006.

GUCCIONE, A. A. Fisioterapia geriátrica. 2. ed. Rio de Janeiro: Guanabara Koogan, 2000. 
GUEDES, J.M; SILVEIRA, R. C. R. Análise da capacidade funcional da população geriátrica institucionalizada na cidade de Passo Fundo - RS. Rev Bras Cien Envel Hum, v.1, n. 2, jul./dez. 2004 HAMILTON, I. S. A psicologia do envelhecimento: uma introdução. Porto Alegre: Artmed, 2002. MATSUDO, S. M. Avaliação do idoso: física e funcional. São Caetano do Sul: Phorte, 2005. MEIRELLES, M. Atividade física na terceira idade. Rio de Janeiro: Sprint, 1997. MINCATO, P. C; FREITAS, C. R. Qualidade de vida dos idosos residentes em instituições asilares da cidade de Caxias do Sul-RS. Rev Bras Cien Envel Hum, v. 4, n. 1, p.127-138, jan./jun. 2007.

OLIVEIRA, C. R. M; SOUZA, C.S; FREITAS, T. M. Idosos e família: asilo ou casa. Disponível em: psicologia.com.pt.

OLIVEIRA, D. V.; SILVA, E.V; KRAVCHYCHYN, C. Prevalência de obesidade em mulheres na terceira idade praticantes de hidroginástica nas academias da cidade de Maringá/PR. In: ENCONTRO INTERNACIONAL DE PRODUÇÃO CIENTÍFICA DO CESUMAR, 5., Maringá, 2007. Anais... Maringá: Cesumar, 2007.

QUADROS JÚNIOR, A.C. et al. Estudo do nível de atividade física, independência funcional e estado cognitivo de idosos institucionalizados: análise por gênero. J Bras Biom, p. 39-50, fev. 2008.

REBELATTO, J. R; MORELLI, J. G. S. Fisioterapia geriátrica: a prática da assistência ao idoso. São Paulo: Manole, 2007.

REIS, L. A.; TORRES, G. V.; REIS, L. A. Características sócio-demográficas de saúde de idosos de uma instituição do município de Jequeié/BA. Rev Espaço Saúde, v .9, n. 2, p. 39-46, jun. 2008.

ROSA, T.E.C. et al. Fatores determinantes da capacidade funcional entre idosos. Rev Bras Saúde Pública, v. 37, n. 1, fev. 2003.

TEIXEIRA, I. N. D. O; NERI, A. L. Envelhecimento bem sucedido: uma meta no curso da vida. Rev Psicol USP, v. 19, n. 1, março 2008.

UENO, L. M. A Influência da atividade física na capacidade funcional: Envelhecimento. Rev Bras Ativ Fis Saúde, v. 4, n. 1, p. 57-68, 1999

YAMAMOTO, A; DIOGO, M.J D. Os idosos e as instituições asilares do município de Campinas-SP. Rev Latino Amer Enferm, Ribeirão Preto, v. 10, n. 5, set./out. 2002.

ZIMERMAN, G. Velhice: Aspectos Biopsicossociais. São Paulo: Artes Médicas, 2000. 


\section{Daniel Vicentini de Oliveira}

Centro Universitário de Maringá

\section{Terezinha Gomes Faria}

Centro Universitário de Maringá

\section{Rosangela Cocco Morales}

\section{Centro Universitário de Maringá}

\section{Marcia Regina Benedeti \\ Centro Universitário de Maringá}

\section{Referência do artigo}

\section{ABNT}

OLIVEIRA, D. V. et al. Análise da capacidade funcional de idosos institucionalizados a partir da autoavaliação. Conexões, v. 7, n. 2, p. 79-96, 2009.

\section{APA}

Oliveira, D. V., Benedetti, M. R., Morales, R. C., Faria, T. G. (2009) Análise da capacidade funcional de idosos institucionalizados a partir da auto-avaliação. Conexões, 7(2),79-96.

\section{VANCOUVER}

Oliveira DV, Benedetti MR, Morales RC, Faria TG. Histórias esquecidas do esporte. Conexões, 2009; 7(2): 79-96.

Recebido em: 04/12/2008

Aceito para publicação em: jun. 2009 